\title{
Arthroscopic-Guided Balloon Tibioplasty in Schatzker III Tibial Plateau Fracture
}

\author{
Fabrizio Cuzzocrea ${ }^{1} \quad$ Eugenio Jannelli ${ }^{1} \quad$ Alessandro Ivone $^{1} \quad$ Simone Perelli $^{1} \quad$ Alberto Fioruzzi $^{1}$ \\ Matteo Ghiara ${ }^{1}$ Giacomo Zanon ${ }^{1}$ Francesco Benazzo ${ }^{1}$ \\ 1 Department of Orthopaedics and Traumatology, University of Pavia, \\ IRCCS Fondazione Policlinico San Matteo, Pavia, Italy \\ Joints 2018;6:220-227. \\ Address for correspondence Eugenio Jannelli, MD, Clinica di \\ Ortopedia e Traumatologia, Fondazione IRCCS Policlinico San Matteo, \\ Università degli studi di Pavia, Pzz.le Golgi,19, Padiglione n.29, \\ Pavia 27100, Italy (e-mail: eugenio.jannelli@libero.it).
}

\begin{abstract}
Keywords

- tibioplasty

- arthroscopy

- fixation

- balloon

Purpose The study aims to present the results at a mean 28-months follow-up of arthroscopic-guided balloon tibioplasty and to spot some technical tricks and some practice using tools and materials.

Methods The study relates to six patients with tibial plateau fractures type Schatzker III with tibial plateau depression more than $4 \mathrm{~mm}$ at preoperative computed tomography scan (CT-scan). The follow-up period ranged from 22 to 33 months, with a mean of 28 months. No patients were lost to follow-up. The patients were evaluated clinically using the Rasmussen score system and Lysholm score systems at 6 to 12 and 24 months, postoperatively. Radiographic evaluations (standard X-rays) were done preoperatively at 1,3 , and 12 months postoperatively while a CT-scan with 3D reconstruction was performed preoperatively, at the first day and 6 months, postoperatively.

Results The mean Rasmussen clinical score at 6 months postoperatively was 26.3 while at 1-year postoperatively the mean Rasmussen clinical score was 28.33. At 2-year postoperatively the mean Rasmussen clinical score was 28.83 . Statistically significant difference was found in 6-months and 2 -years results $(p<0.05)$. CT-scan achieved the first postoperative day showed the recovery of approximately $70 \%$ of the area of the interested tibial plateau, restoring of the joint surface without articular bone free fragments.

Conclusion The described surgical procedure, if correctly performed with proper indications (Schatzker III), respect the principles mentioned above and the clinical and radiological results confirm our purpose.

Level of Evidence This is a therapeutic case series, level IV study.
\end{abstract}

\section{Introduction}

Tibial plateau fractures are $1 \%$ of the adult fractures and 5 to $8 \%$ of the lower limb fractures: males are more affected than females. ${ }^{1}$ The mechanism usually involves a varus or valgus loaded with axial forces.

The most frequent fractures are the ones with articular surface depression (Arbeitsgemeinschaft für Osteosynthesefragen/Orthopaedic Trauma Association [AO/OTA] type 41. $\mathrm{B} 2$, Schatzker III) with or without lateral condyle split (AO/
OTA type 41.B3, Schatzker II). Schatzker III are mainly found in elderly and due to osteopenia, whereas Schatzker II are typical in young adults and occur due to high energy trauma. $^{2}$

Surgical treatment is indicated for a depressed area bigger than $1 \mathrm{~cm}^{2}$ and/or more than 10 degree of valgus deformity, evaluated with the knee in extension. ${ }^{3}$

The standard treatment of tibial plateau fracture is open reduction and internal fixation (ORIF) with plates and screws, received

January 19, 2018

accepted

August 6, 2019

published online

October 11, 2019
DOI https://doi.org/

10.1055/s-0039-1697607.

ISSN 2282-4324.
Copyright @ 2018 Georg Thieme Verlag

KG Stuttgart · New York

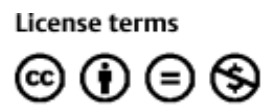


with or without the filling of bone defect with different bony substitutes. This kind of treatment requires surgical injuries to soft tissues which are most often undamaged. Furthermore, the articular surface restoration is difficult to achieve from below and sometimes also an overcorrection can be seen as an intraarticular prominence of the cartilage.

Nowadays, the patient's functional level is more and more demanding, so the attempt is to spare the soft tissues and to minimize the surgical approach also with the arthroscopically assisted techniques.

Sparing soft tissues can enhance the bone callus formation, limiting the periosteal devascularization and the complications related to the surgical approach.

Caspari et $\mathrm{al}^{4}$ and Jennings, ${ }^{5}$ in the 1980 s, were the first to describe arthroscopically assisted osteosynthesis; since then, other mini-invasive techniques have been developed.

This study aims to describe our technique, consisting of arthroscopic reduction and internal fixation (ARIF) with ballooning, underlining technical aspects, and reporting the clinical and radiographic results of our patients with a mean follow-up of 2 years.

\section{Methods}

The study relates to six patients (four males and two females) with tibial plateau fracture type Schatzker III with tibial plateau depression more than $4 \mathrm{~mm}$ at preoperative computed tomography scan (CT-scan), treated between February 2014 and January 2016. The mean age of group was 46 years (36-55 years).

Exclusion criteria were patients under the age of 20 , posterior tibial plateau comminutions, and a compartment syndrome before surgery.

The mean time between the day of admission and surgery was 4 days (range 2-10 days), and the timing of the operation was influenced by the patient's general health status (cardiopulmonary diseases and anticoagulant therapy) and the soft-tissue envelope conditions, particularly, significant edema and skin blisters.

A preoperative CT-scan study allowed the fracture classification to evaluate comminution and the precise site and the extension of the articular surface damage; this information was essential for the placement of balloon guidewires to reduce and to fix the bony defect.

The surgical technique was an ARIF tibioplasty performed with expansive cranial balloon filled with tricalcium phosphate and percutaneous screw stabilization. All patients were treated by the same surgeon.

\section{Treatment}

In this technique, a kyphoplasty balloon was used for the reduction of the fracture and a tricalcium phosphate filler to fulfill the bone defect and to stimulate the healing.

On a radiotransparent surgical table, the patient was in a supine position. A tourniquet was used, and two holders were set at the foot and in the lateral region of the thigh. The knee had to be free to flex until 90 degree. Epidural anesthesia and preoperative assessment of the knee were usually performed.
The first surgical procedure was the arthroscopy with anteromedial and anterolateral portals. We usually used a load lower than $20 \mathrm{~mm} \mathrm{Hg}$. In the arthroscopic procedure, different aims were possible: articular cleaning, ligaments, meniscal and cartilage assessment; also far from the fracture site, were essential preliminary acts, but above all, with arthroscopy performed a proper evaluation of the quality of the fracture reduction was obtained through external manipulations.

The second procedure was the setting of two K wires to stabilize the fracture at the anterior and posterior edges and to control the extension right below the subsiding, where the balloon was set. For a correct $\mathrm{K}$ wires positioning, the preoperative CT-scan was crucial to know the morphology of the subsidence. $\mathrm{K}$ wires and osteointroducers had to be perfectly parallel to the joint line, and so also the natural slope of the tibia and other congenital or post-traumatic deformities had to be considered. A further fluoroscopic control of the proper placement of $\mathrm{K}$ wires in both anteroposterior and lateral views was performed.

The problem of the expansive direction of the inflated balloon was solved putting into the place more than two $\mathrm{K}$ wires realizing a wall and thus avoiding the caudal expansion of the balloon.

The balloon allowed only a cranial expansion, avoiding the significant impact on the trabeculae below the fracture, and thus the metaphyseal filler spreading was avoided. Furthermore, this polar expansion could be achieved by less than $17 \mathrm{~atm}$ pressure which is lower than the pressure necessary for the circumferential expansion. ${ }^{6}$

Once the position of the balloon was controlled, the pressure could be progressively increased using a contrast liquid, and the possible asymmetry of balloon expansion under fluoroscopy could be assessed.

The arthroscopic evaluation was performed to have an intra-articular control of cartilage layer and to see if the balloon had to be moved. Using a probe under arthroscopic assistance, we could also adjust the cartilage layer, especially in multifragmentary fractures. In large fractures, we could move the balloon in the peripheral depression and check its proper position under arthroscopy, and if necessary, we could set further osteointroducers.

Once a satisfying reduction was achieved, a fluoroscopic control was used to assess the good position obtained of the subchondral bony layer. Thus the balloon could be removed.

The cavity obtained using the balloon was stabilized by compression of the trabeculae, and so it could be filled by tricalcium phosphate, which secures the fracture and acts as a mechanical support.

The filler was introduced by the trocar, and its volume was deduced by the contrast liquid volume: $6 \mathrm{~mL}$ of tricalcium phosphate was usually used, and a new fluoroscopic and arthroscopic evaluation was suggested after the filling. The filler was transformed into calcium apatite in 12 minutes. Then the final synthesis was performed putting $7.3-\mathrm{mm}$ cannulated screws on the previously set $\mathrm{K}$ wires. These screws had to be placed below the filler (-Fig. 1).

The early mobilization was possible from the first day, $50 \%$ of the weight bearing on the affected side was allowed after 


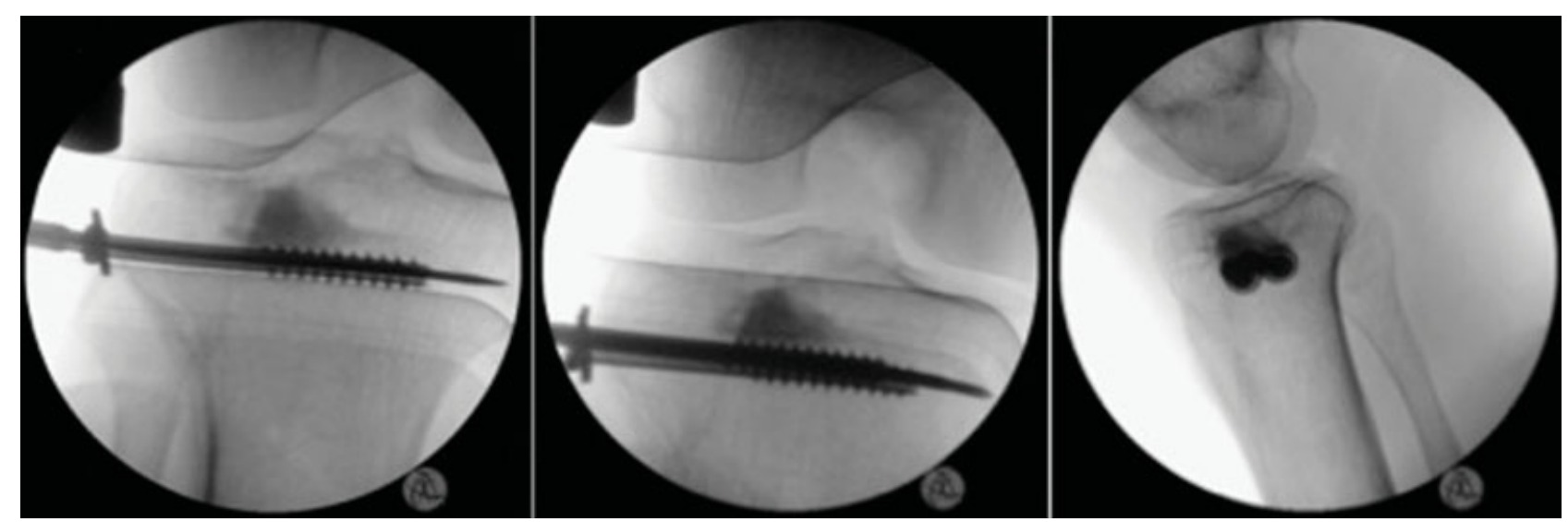

Fig. 1 Intraoperative fluoroscopic control of the proper placement of the screw.

15 days. Complete weight bearing was allowed after 1 month. When the postoperative pain reduced, muscular strength exercises were allowed. At 6 months, a CT-scan evaluation was required, and if an excellent muscular strength and ROM were achieved, return to the previous sports activity was allowed.

\section{Outcome Measurements}

The patients were evaluated clinically using the Rasmussen score system ${ }^{7}$ and Lysholm score system ${ }^{8}$ at 6 to 12 and 12 to 24 months, postoperatively. Radiographic evaluations (standard X-rays) were done preoperatively (-Figs. 2 and $\mathbf{3}$ ) and at 1,3 , and 12 months postoperatively while a CT-scan with 3D reconstruction was performed preoperatively (-Fig. 4), at first day and 6 months, postoperatively.

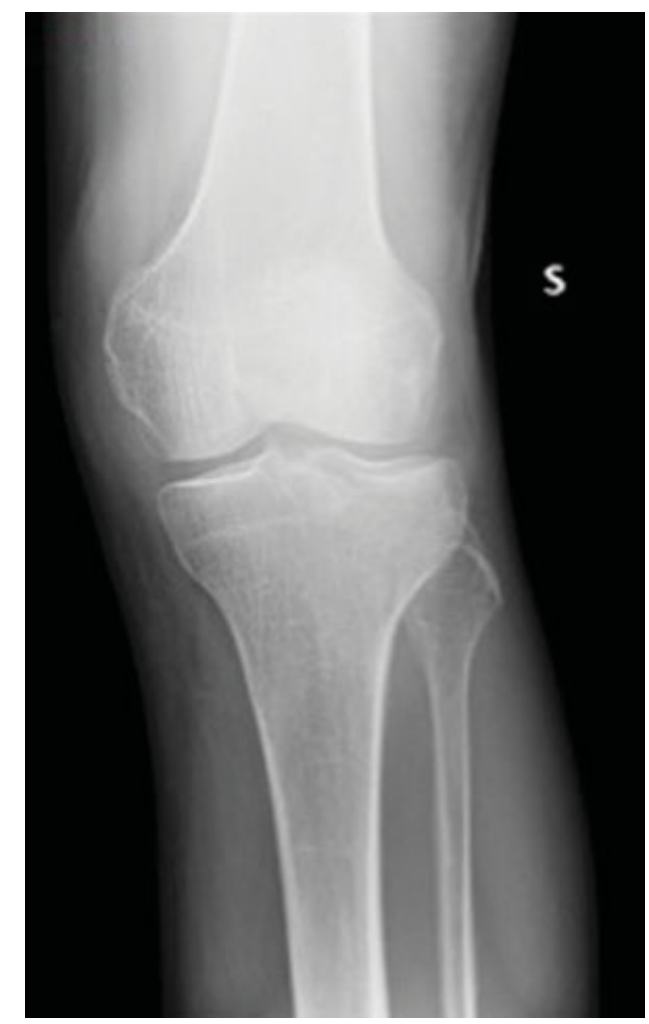

Fig. 2 Preoperative X-ray anteroposterior view.

\section{Statistical Analysis}

The data from this study were analyzed through the use of the SPSS statistics software program (SPSS Inc. Chicago, United States). A t-test was used for normally distributed, continuous variables.

A $p$-value $<0.05$ was considered statistically significant. Descriptive statistics are presented as mean (range).

\section{Results}

The follow-up period ranged from 22 to 33 months, with a mean of 28 months. No patients were lost to follow-up.

The mean Rasmussen clinical score at 6-months postoperatively was 26.3 (range 22-28). Walking, motion, and

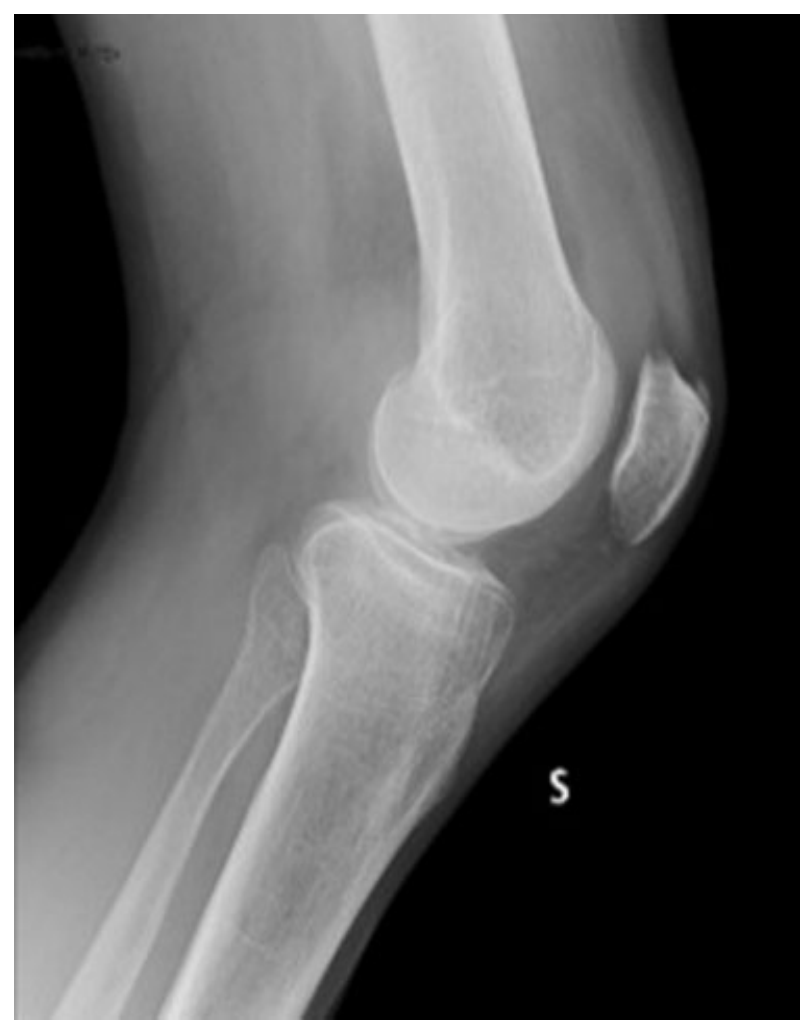

Fig. 3 Preoperative X-ray lateral view. 

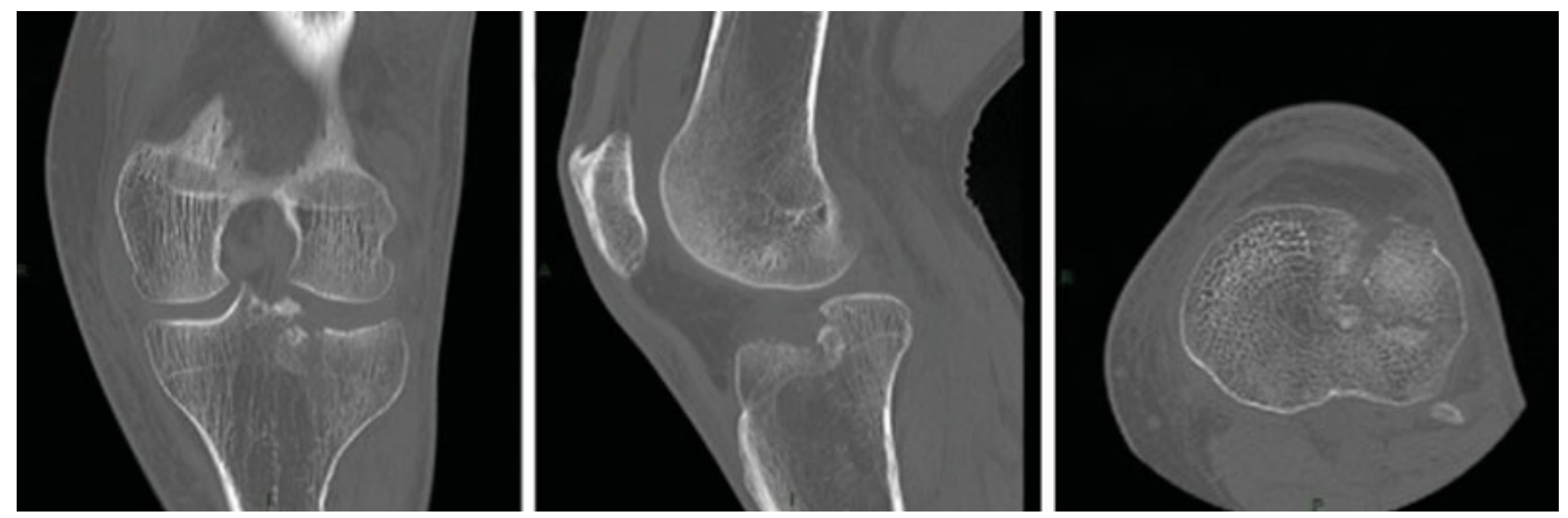

Fig. 4 Preoperative CT-scan shows tibial plateau depression more than $4 \mathrm{~mm}$.

stability were good or excellent in five cases. Only one patient reported occasional and local pain and poor walking ability, without stiffness and articular laxity. In this patient, at 6months CT-scan, an osteolytic area was present at the tip of the screws on the head of the fibula, because of the too long screw (-Figs. 5 and $\mathbf{6}$ ), so we planned surgical removal.

At 1-year postoperatively, the mean Rasmussen clinical score was 28.33 (range 25-30). All patients showed improvement in all fields studied. The worst results were reported in the patients who underwent screws removal, compatible with the recent surgery. No statistically significant difference was found between 6-months and 1-year results $(p>0.05)$.

At 2-year postoperatively, the mean Rasmussen clinical score was 28.83 (28-30). All patient presented excellent results in all categories, with an important reduction of the outcomes difference at 2 years. No statistically significant difference was found between 1-year and 2-years results $(p>0.05)$ but a significant difference was found between 6months and 2-years results $(p<0.05)(-$ Table 1$)$. They had an excellent medium-term outcome and were free of symptoms with a full active range of motion of knee (0140 degree).

We observed the same trend in Lysholm Knee score (-Table 2), in which at 6-month follow-up the mean was 90.2 (range 85-95) with excellent results in five of six patients. At 12-months follow-up, the mean Lysholm Knee score was 94.8 (range 85-100), with excellent results and significant variability because of the patient who underwent screw removal. As a matter of fact, at 24-months follow-up the mean Lysholm Knee score was 97.3 (range 96-100), and all the results were excellent.

In Lysholm Knee score also there was no significant difference in 6-months/1-year results and 1-year/2-years results $(p>0.05)$ but with an important difference in 6months/2-years results $(p<0.001)$.

CT-scan achieved the first postoperative day showed the recovery of approximately $70 \%$ of the area of the interested tibial plateau, restoring of the joint surface without articular bone free fragments. The control CT-scan, performed at 6months postoperatively showed the same radiological aspects in five of six patients ( - Fig. 7), except in the patient treated with surgical screw removal.
Planned radiographic controls demonstrated a maintained anatomic articular reduction ( - Fig. 8), without subsidence or screw mobilization.

At last radiographic controls, no radiological sign of tricalcium phosphate reabsorption was found.

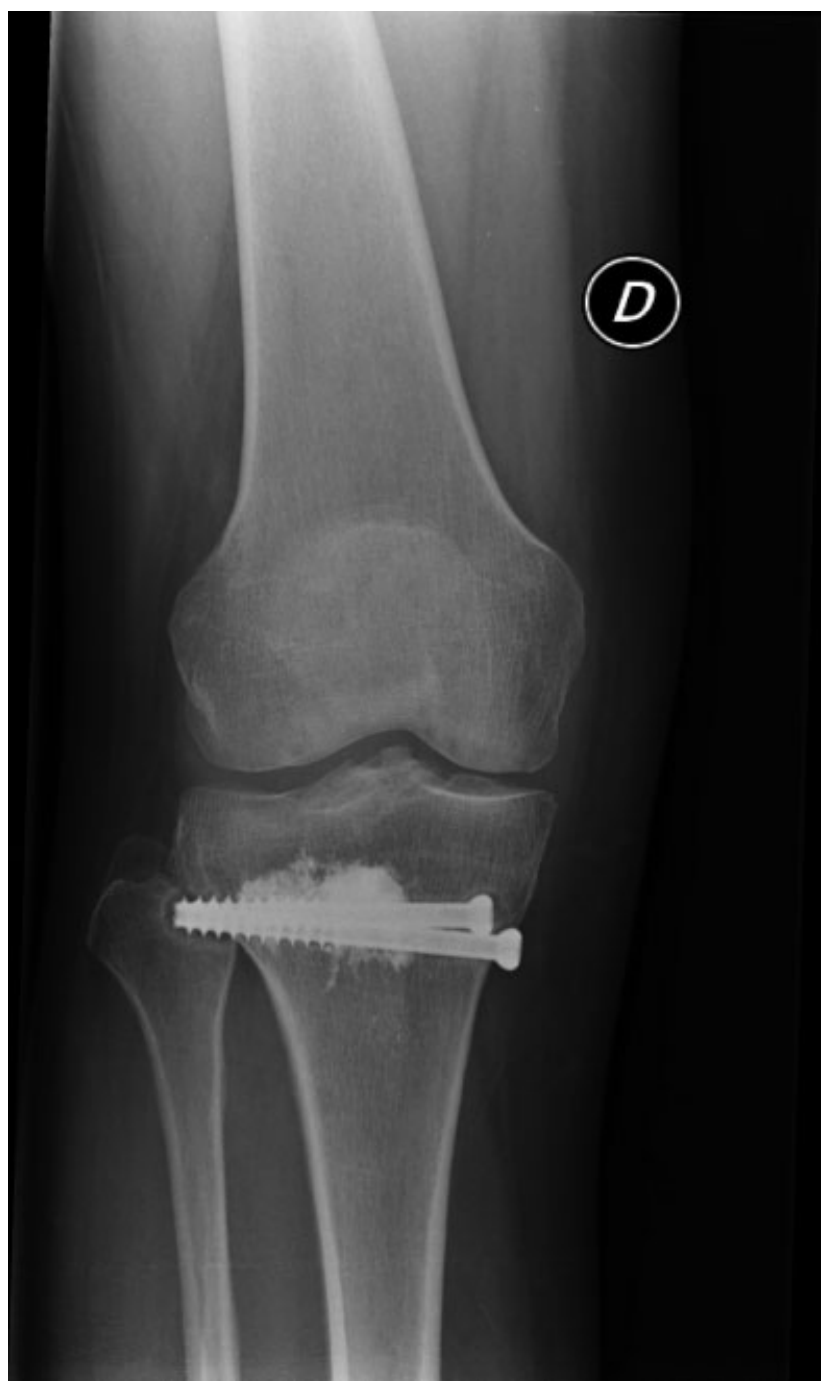

Fig. 5 Control X-ray at 6-months follow-up: it presented an osteolytic area at the tip of the screws. 


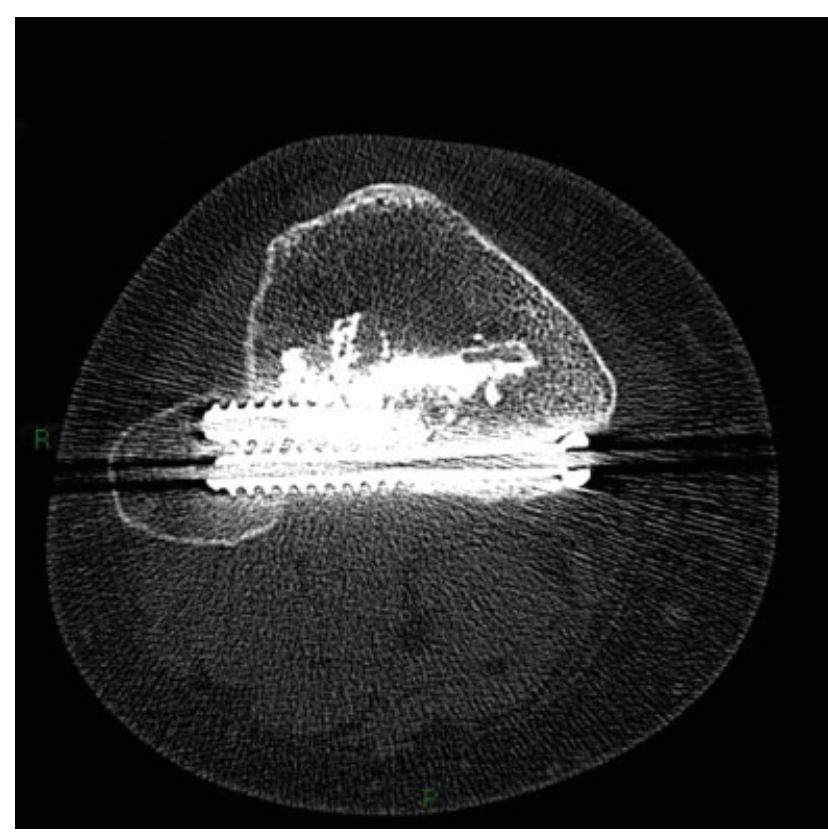

Fig. 6 Control CT-scan at 6-months follow-up: the osteolytic area was better visible with axial scans.

Table 1 Rasmussen clinical score at 6 to 12 and 12 to 24 months follow-up

\begin{tabular}{|l|l|l|l|}
\hline Patients & 6-months & 12-months & 24-months \\
\hline $\mathbf{1}$ & 27 & 29 & 29 \\
\hline 2 & 27 & 29 & 29 \\
\hline 3 & 28 & 30 & 30 \\
\hline 4 & 28 & 29 & 29 \\
\hline 5 & 26 & 28 & 28 \\
\hline 6 & 22 & 25 & 28 \\
\hline
\end{tabular}

Table 2 Lysholm Knee score at 6 to 12 and 12 to 24 months follow-up

\begin{tabular}{|l|l|l|l|}
\hline Patients & 6-months & 12-months & 24-months \\
\hline $\mathbf{1}$ & 90 & 95 & 96 \\
\hline $\mathbf{2}$ & 95 & 100 & 100 \\
\hline $\mathbf{3}$ & 90 & 95 & 96 \\
\hline $\mathbf{4}$ & 91 & 99 & 100 \\
\hline $\mathbf{5}$ & 90 & 95 & 96 \\
\hline $\mathbf{6}$ & 85 & 85 & 96 \\
\hline
\end{tabular}

\section{Discussion}

This study presents the results referring to the treatment of tibial plateau fracture type Schatzker III by balloon tibioplasty arthroscopically assisted. In our series of patients the reduction of the fracture can be obtained by this miniinvasive approach in all cases and consequently the joint cartilage surface can be restored in a large area of the fracture. Clinical and functional scores showed excellent results at the medium follow-up; rehabilitation started the first day after surgery with a fast recovery of full range of motion and restoring of weightbearing after 2 weeks. Radiological controls (CT-scan and radiographs) confirmed the durable fracture reduction and the absence of post-traumatic arthritis. Only one patient reported occasional pain related to screws length and position.

The goals of surgical treatment of these injuries are restoration of the joint surface, tibial alignment, fast rehabilitation to prevent stiffness of the knee, and avoidance of posttraumatic arthritis. Traditionally, these fractures can be treated by open reduction (by a window into the tibial bone) and internal fixation by plate, locking screws and bone graft. Sometimes a mini-arthrotomy or arthroscopy can be indicated to confirm the articular surface restoration. Complications of ORIF surgery can be muscular damage, postoperative pain and swelling, infection, operation time, scarring, and damage to the blood supply to the periosteum. ${ }^{9}$ These evidences encouraged the use of percutaneous reduction with balloon and cement augmentation in tibial plateau fractures, a technique derived from vertebral surgery but also used successfully in maxillofacial surgery and for the treatment of calcaneus, ${ }^{10}$ cuboid, ${ }^{11}$ distal radius, ${ }^{12}$ and acetabular $^{13}$. Reiley, ${ }^{14}$ in 2003, first described this technique for the tibial plateau.

Pizanis et $\mathrm{al}^{15}$ presented a case series of five patients treated by balloon tibioplasty and plate fixation; the results at 12 to 36-months follow-up showed no loss of reduction and no development of post-traumatic arthritis. The functional evaluation with the Rasmussen and Lysholm scores yielded excellent results (respectively 28-30 points and 95-100 points), so their results are in agreement with our findings. The authors recommend the use of a lateral plate to avoid an unwanted breach through the lateral cortex during fracture reduction and the use of $\mathrm{K}$ wire under the fracture to favor balloon direction during fracture reduction.

Hahnhaussen et al $^{16}$ presented a case report on a female of 51 year-old with a depressed lateral tibial plateau fracture (Schatzker III) managed by balloon tibioplasty and plate fixation; the follow-up at 1 year showed excellent clinical outcome and a maintained long-term reduction and fixation on radiographs.

Doria et $\mathrm{al}^{9}$ performed a randomized multicentric trial on 28 patients with lateral tibial plateau fracture (Schatzker II: 3 cases-Schatzker III 25 cases). The patients were randomized in two groups: fracture reduction using balloon tibioplasty (14 patients) and traditional technique (14 patients). Only in one case the surgeons added a plate to the tibioplasty; in the other case tibioplasty was performed alone. They showed that mean Rasmussen score was higher in tibioplasty group (28.9 vs. 26.1, 1-year follow-up) and that there was no loss of articular reduction at 6 -weeks follow-up in this group. Postoperative CT-scan demonstrated the recovery of approximately $75 \%$ of the area of the tibial lateral plateau joint surface. They concluded that this new technique leads to better results and less surgical trauma than traditional 

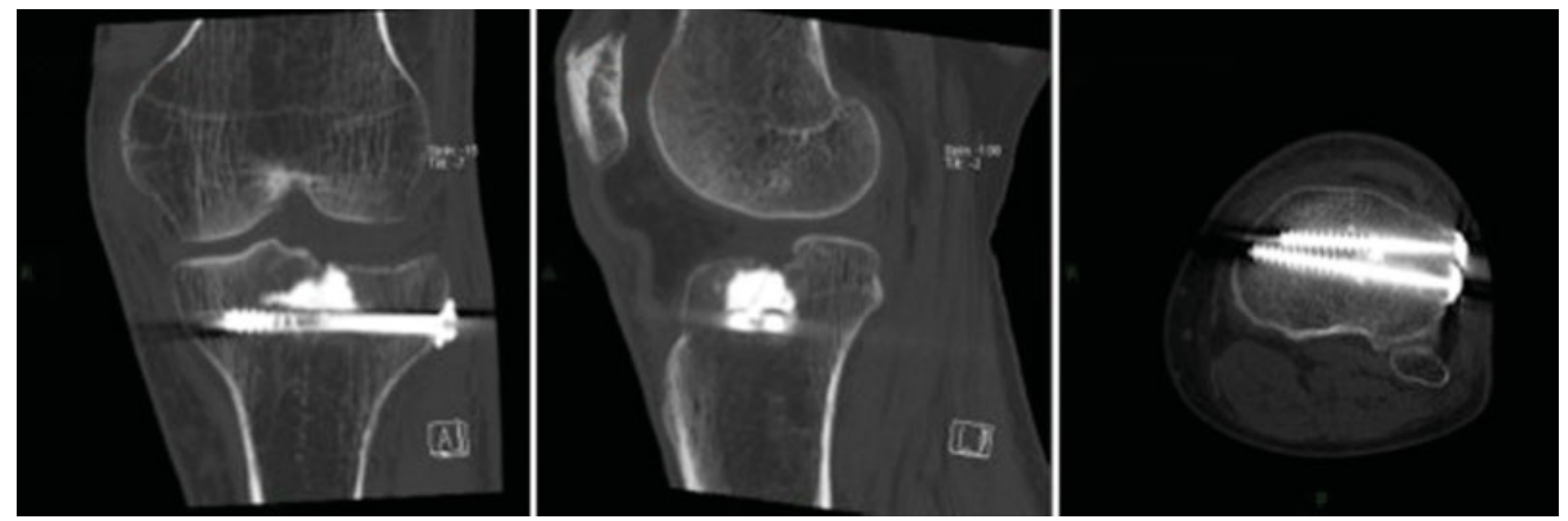

Fig. 7 Control CT-scan at 6-months follow-up shows the recovery of approximately $70 \%$ of the area of the interested tibial plateau, restoring of the joint surface without articular bone free fragments.

surgery; the only drawback is the higher cost due to instruments.

Ollivier et al $^{17}$ presented a paper on 20 patients suffering from lateral tibial plateau fracture (Schatzker II and III), managed with inflation tibioplasty and restorable calcium phosphate bone substitute injection. At 1-year follow-up, they observed good results in terms of pain, daily living activities, and quality of life without occurrence of perioperative complications. The step-off depression of lateral tibial plateau improved from 10 to $2-\mathrm{mm}$, postoperatively. They concluded that the procedure is safe and it allows to obtain a high rate of anatomic reduction.

Mauffrey et $\mathrm{al}^{18}$ in an observational retrospective study on 20 patients showed the most frequent complications related to this technique: burst balloon, extrusion of cement in soft tissue, no reduction of depressed articular fragment, and intra-articular penetration of the cement. In another paper, Mauffrey et al ${ }^{19}$ highlighted the pearls and the pitfall of this surgical technique. Balloon tibioplasty is superior to a metal tamp to reduce articular and comminuted fracture, but a complete cortical ring is mandatory for achieving articular reduction. So in the presence of a cortical split the surgeon must close the split to create a cortical ring before balloon inflation to avoid "the trap-door effect" and the risk of an insufficient reduction of the articular fragment. Generally a volume of $4 \mathrm{~mL}$ is required to elevate a tibial plateau fracture, but the surgeon must give attention to pressure reading because in a nonfractured area the pressure increases rapidly with the risk of balloon breakage. Finally, in the presence of large defect and depression, a second balloon can be required to obtain good reduction.

In literature there is no agreement regarding the ideal fixation associated with tibioplasty; the procedure can be performed alone ${ }^{9}$ or in association with screws or plate placement. In our experience (Schatzker III fracture), the use of screws provided a support to the fracture reduction

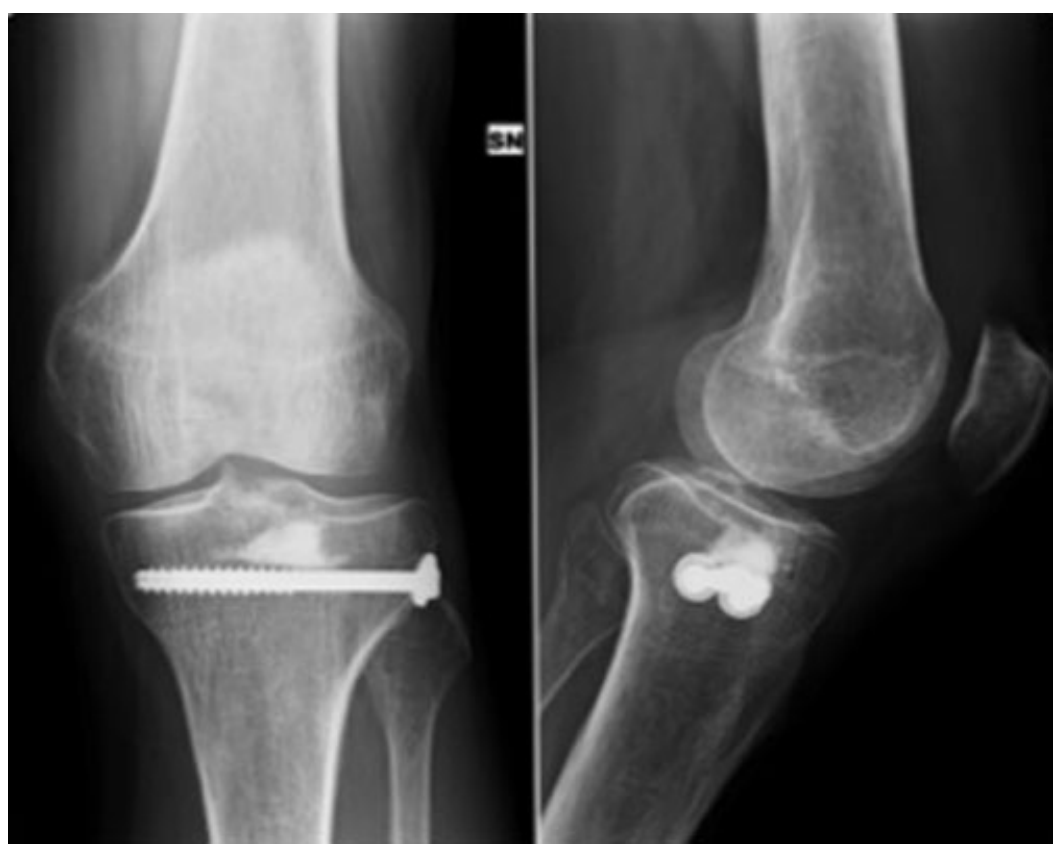

Fig. 8 Control X-ray at 12-months follow-up. 
and allowed quick postoperative weightbearing. The use of plate is indicated in case of Schatzker II fracture because there is split interesting cortical bone, especially in the presence of a porotic bone. In young patients we recommend the use of screws to preserve periosteal vascularization and improve bone healing.

Belaid et $\mathrm{al}^{20}$ presented a finite element analysis on stress distribution in the tibial plateau stabilized by balloon osteoplasty to determine the influence of cement filling. They concluded that the cement injection can increase implant stability, preserve fracture reduction, and produce a uniform load distribution.

Simpson and Keating ${ }^{21}$ showed calcium phosphate properties. The potential stimulation of the bone healing is the same as that of the bone impaction grafting, but calcium phosphate allows a quicker surgical time and a better reduction at 1-year follow-up. It does not need an exothermic reaction as required for the acrylic cement, avoiding thermic cellular damage and saving its osteoinductive properties.

In literature some authors use only fluoroscopy ${ }^{9}$ to evaluate intraoperative fracture reduction; other authors combine fluoroscopy and arthroscopy. ${ }^{15,19}$ In our experience, arthroscopy allows a better vision of the whole joint, especially of cartilage surface restoration, as well as the possibility to treat any intra-articular lesions and hematoma removal. However, the surgeon must pay attention to arthroscopic pump pressure to avoid compartment syndrome due to the extra-articular spreading of liquid. The limit of pressure allowed is not defined and the suggestion is to use the lowest possible pressure level. Dall'Oca et $\mathrm{al}^{22}$ compared ARIF treatment with traditional ORIF in 100 patients and they showed that ARIF technique may increase clinical outcome in tibial plateau fractures (Schatzker II, III, and IV type). The patients showed better range of motion, less postoperative pain, and less incidence of infection. In type III fracture, they found a high of incidence of lateral meniscal tears, suggesting that ARIF also allowed to identify and to treat associated articular lesions.

Wang et $\mathrm{al}^{23}$ will conduct a blinded randomized controlled trial on 80 patients managed with arthroscopicassisted balloon tibioplasty and open reduction and internal fixation; the outcome measure at 2-years follow-up will be clinical and radiological score, quality of reduction, intraoperative blood loss, surgical duration, postoperative pain, hospitalization, reoperation, and post-traumatic arthritis development. So more information about this novel technique can be understood by this study.

There are limitations to our study: the number of patient is less, there is no control group, and the follow-up at 2 years cannot exclude certainly the development of post-traumatic arthritis.

Based on our results and literature considerations, we can conclude that balloon tibioplasty allows to obtain excellent clinical and radiological results if surgery is performed with proper indications related to Schatzker classification (type III in our experience). Articular surface restoration and fracture reduction can be obtained by this mini-invasive technique.
The procedure is safe, but the surgeon has to follow standardized steps and surgical tricks to avoid complications. Anyway, randomized controlled studies and a broader cohort with longer follow-up are needed to confirm these encouraging data.

\section{Conflict of Interest}

None declared.

\section{References}

1 Petersen W, Zantop T, Raschke M. Tibiakopffraktur. Unfallchirurg 2006;109(03):219-232

2 Marsh JL, Slongo TF, Agel J, et al. Fracture and dislocation classification compendium-2007: Orthopaedic Trauma Association classification, database, and outcomes committee. JOrthop Trauma 2007;21(10, Suppl):S1-S133

3 Watson JT, Schatzker J. Tibial plateau fractures. In: Browner BD, ed. Skeletal Trauma: Basic Science, Management, and Reconstruction. 3rd ed. Philadelphia, PA: Saunders; 2003:2047-2130

4 Caspari RB, Hutton PM, Whipple TL, Meyers JF. The role of arthroscopy in the management of tibial plateau fractures. Arthroscopy 1985;1(02):76-82

5 Jennings JE. Arthroscopic management of tibial plateau fractures. Arthroscopy 1985;1(03):160-168

6 Broome B, Mauffrey C, Statton J, Voor M, Seligson D. Inflation osteoplasty: in vitro evaluation of a new technique for reducing depressed intra-articular fractures of the tibial plateau and distal radius. J Orthop Traumatol 2012;13(02):89-95

7 Rasmussen PS. Tibial condylar fractures. Impairment of knee joint stability as an indication for surgical treatment. J Bone Joint Surg Am 1973;55(07):1331-1350

8 Tegner Y, Lysholm J. Rating systems in the evaluation of knee ligament injuries. Clin Orthop Relat Res 1985;(198):43-49

9 Doria C, Balsano M, Spiga M, Mosele GR, Puddu L, Caggiari G. Tibioplasty, a new technique in the management of tibial plateau fracture: a multicentric experience review. J Orthop 2017;14(01): 176-181

10 Bano A, Pasku D, Karantanas A, Alpantaki K, Souvatzis X, Katonis P. Intra-articular calcaneal fracture: closed reduction and balloonassisted augmentation with calcium phosphate cement: a case report. Cases J 2009;2:9290

11 Heim KA, Sullivan C, Parekh SG. Cuboid reduction and fixation using a kyphoplasty balloon: a case report. Foot Ankle Int 2008;29 (11):1154-1157

12 Iida K, Sudo A, Ishiguro S. Clinical and radiological results of calcium phosphate cement-assisted balloon osteoplasty for Colles' fractures in osteoporotic senile female patients. JOrthop Sci 2010;15(02):204-209

13 König B, Khodadadyan C, Schäffler A, Pflugmacher R, Stöckle U. [Percutaneously navigated balloon fracture reduction in a displaced acetabular fracture]. Unfallchirurg 2007;110(12): 1072-1075

14 Reiley MA. Abstracts from the 8th annual meeting of the international society for fracture repair. Number 800 Percutaneous balloon-plasty technique. J Orthop Trauma 2003;17:141-163

15 Pizanis A, Garcia P, Pohlemann T, Burkhardt M. Balloon tibioplasty: a useful tool for reduction of tibial plateau depression fractures. J Orthop Trauma 2012;26(07):e88-e93

16 Hahnhaussen J, Hak DJ, Weckbach S, Heiney JP, Stahel PF. Percutaneous inflation osteoplasty for indirect reduction of depressed tibial plateau fractures. Orthopedics 2012;35(09): 768-772

17 Ollivier M, Turati M, Munier M, Lunebourg A, Argenson JN, Parratte S. Balloon tibioplasty for reduction of depressed tibial plateau fractures: preliminary radiographic and clinical results. Int Orthop 2016;40(09):1961-1966 
18 Mauffrey C, Fader R, Hammerberg EM, Hak DJ, Stahel PF. Incidence and pattern of technical complications in balloonguided osteoplasty for depressed tibial plateau fractures: a pilot study in 20 consecutive patients. Patient Saf Surg 2013;7 (01):8

19 Mauffrey C, Roberts G, Cuellar DO, Herbert B, Hak D. Balloon tibioplasty: pearls and pitfalls. J Knee Surg 2014;27(01):31-37

20 Belaid D, Vendeuvre T, Bouchoucha A, et al. Utility of cement injection to stabilize split-depression tibial plateau fracture by minimally invasive methods: a finite element analysis. Clin Biomech (Bristol, Avon) 2018;56:27-35
21 Simpson D, Keating JF. Outcome of tibial plateau fractures managed with calcium phosphate cement. Injury 2004;35(09): 913-918

22 Dall'oca C, Maluta T, Lavini F, Bondi M, Micheloni GM, Bartolozzi P. Tibial plateau fractures: compared outcomes between ARIF and ORIF. Strateg Trauma Limb Reconstr 2012;7(03):163-175

23 Wang JQ Jiang BJ, Guo WJ, Zhang WJ, Li AB, Zhao YM. Arthroscopic-assisted balloon tibioplasty versus open reduction internal fixation (ORIF) for treatment of Schatzker II-IV tibial plateau fractures: study protocol of a randomised controlled trial. BMJ Open 2018;8(08):e021667 